\title{
Téoros
}

Revue de recherche en tourisme

\section{Opérationnaliser le développement durable en contexte écotouristique : quels critères ?}

\section{Manu Tranquard et Christiane Gagnon}

Volume 31, numéro 2, 2012

URI : https://id.erudit.org/iderudit/1020773ar

DOI : https://doi.org/10.7202/1020773ar

Aller au sommaire du numéro

Éditeur(s)

Université du Québec à Montréal

ISSN

0712-8657 (imprimé)

1923-2705 (numérique)

Découvrir la revue

\section{Citer cet article}

Tranquard, M. \& Gagnon, C. (2012). Opérationnaliser le développement durable en contexte écotouristique : quels critères ? Téoros, 31(2), 72-83.

https://doi.org/10.7202/1020773ar
Résumé de l'article

L’usage du concept de développement durable est actuellement particulièrement répandu dans le secteur touristique. Tout projet d'envergure tend désormais à intégrer dans sa planification ou dans son cahier des charges la recherche des normes et principes de développement durable. Une difficulté tient cependant à la distance qui subsiste entre ce concept et les moyens employés pour y parvenir et l'évaluer. Plusieurs études sectorielles récentes soulignent ainsi la complexité de la mise en application des principes du développement durable dans le domaine touristique. Cette situation tiendrait notamment au relatif défaut de connaissances des fondements théoriques de ce concept de la part des gestionnaires privés et publics et des parties prenantes. Ce constat est par ailleurs d'autant plus problématique dans le cas spécifique de l'écotourisme, car celui-ci repose sur une application rigoureuse des principes du développement durable, notamment pour la dimension environnementale.

L'objet de la présente analyse est d'identifier les critères de la durabilité écotouristique, soit les critères devant être opérationnalisés dans le cadre d'activités écotouristiques afin de favoriser le développement durable des territoires. Au-delà de leur recensement, il s'agit d'évaluer s'il existe une hiérarchisation de certains de ces critères, notamment s'agissant de la dimension environnementale du développement durable. Cette hiérarchisation peut permettre d'optimiser une démarche d'implantation lors de l'élaboration des projets écotouristiques.
Ce document est protégé par la loi sur le droit d'auteur. L'utilisation des services d’Érudit (y compris la reproduction) est assujettie à sa politique d'utilisation que vous pouvez consulter en ligne.

https://apropos.erudit.org/fr/usagers/politique-dutilisation/ 


\title{
Opérationnaliser le développement durable en contexte écotouristique : quels critères?
}

\author{
Manu TRANQUARD, LL. M, Doctorant \\ Professeur, Université du Québec à Chicoutimi (UQAC) \\ Laboratoire d'Expertise et de Recherche en Plein Air (LERPA) \\ Manu_Tranquard@uqac.ca \\ Christiane GAGNON, Ph.D. \\ Professeure, Université du Québec à Chicoutimi (UQAC) \\ Responsable de l'axe aménagement et développement durable \\ du Centre de recherche en développement territorial (CRDT) \\ Christiane_Gagnon@uqac.ca
}

RÉSUMÉ: L'usage du concept de développement durable est actuellement particulièrement répandu dans le secteur touristique. Tout projet d'envergure tend désormais à intégrer dans sa planification ou dans son cahier des charges la recherche des normes et principes de développement durable. Une difficulté tient cependant à la distance qui subsiste entre ce concept et les moyens employés pour y parvenir et l'évaluer. Plusieurs études sectorielles récentes soulignent ainsi la complexité de la mise en application des principes du développement durable dans le domaine touristique. Cette situation tiendrait notamment au relatif défaut de connaissances des fondements théoriques de ce concept de la part des gestionnaires privés et publics et des parties prenantes. Ce constat est par ailleurs d'autant plus problématique dans le cas spécifique de l'écotourisme, car celui-ci repose sur une application rigoureuse des principes du développement durable, notamment pour la dimension environnementale.

L'objet de la présente analyse est d'identifier les critères de la durabilité écotouristique, soit les critères devant être opérationnalisés dans le cadre d'activités écotouristiques afin de favoriser le développement durable des territoires. Au-delà de leur recensement, il s'agit d'évaluer s'il existe une hiérarchisation de certains de ces critères, notamment s'agissant de la dimension environnementale du développement durable. Cette hiérarchisation peut permettre d'optimiser une démarche d'implantation lors de l'élaboration des projets écotouristiques.

Mots-clés: Écotourisme, développement durable, environnement, opérationnalisation, critère.

L'écotourisme peut, sous certaines conditions, être le vecteur qui contribue à ce que des activités de tourisme de nature s'inscrivent dans une démarche de développement durable. Paramétrer ces conditions est l'objet d'une étude plus large portant sur l'ingénierie des projets écotouristiques, dont fait partie la présente contribution. L'objectif de cette dernière est d'opérer un relevé des critères contributifs à la durabilité écotouristique tout en insistant sur leur composante environnementale, facteur prioritaire et limitatif de l'écotourisme. Une contribution subséquente traitera quant à elle des méthodes d'implantation de ces critères.

L’application des principes de développement durable au secteur touristique ne serait pas aussi «naturelle» que d'aucuns pourraient l'envisager (Vernon et al., 2005; Clarimont et Vlès, 2008). Des études sectorielles (CQRHT, 1996; Tourisme Québec, 2002,2007) soulignent par exemple cette relative complexité d'application en contexte québécois. Le développement durable y reste souvent au stade de la déclaration d'intention. Sa mise en œuvre se bute à l'hésitation face au caractère aléatoire de sa prédictibilité, au manque de moyens financiers, au déficit de compétences techniques en gestion de projet, mais surtout au manque de connaissances sur ce qu'implique concrètement l'opérationnalisation de ses trois ou quatre piliers et de l'ensemble de leurs paramètres. Il existe à ce titre plusieurs principes de développement durable, selon les contextes nationaux. Au Québec, toutefois, les 16 principes définis par le ministère du Développement durable, de l'Environnement, de la Faune et des Parcs (voir Loi 118) constituent en quelque sorte une grille d'analyse, un effort d'opérationnalisation pour le gouvernement de l'État, tout en recoupant la littérature sur le développement durable.

Selon des chercheurs qui ont abordé la question de l'opérationnalisation du développement durable appliquée au tourisme (Priskin, 2004; Rivard, 2004; Baholet, 2008; Caire 
et Roulet-Caire, 2001), un certain nombre d'outils seraient pertinents : gestion environnementale, mise en œuvre variable, croissance intelligente, initiatives de collaboration, éthique, acceptabilité sociale, reconnaissance du rôle important des touristes, responsabilisation, labellisation des produits, tourisme solidaire. Certains de ces outils visent notamment à contenir un des obstacles du tourisme durable, soit le fait que le tourisme, surtout de masse, soit une activité humaine génératrice d'impacts. Ces impacts, de différents ordres, sont nombreux, importants et complexes (Tranquard, 2010). Or, si les effets socio-économiques, culturels et sanitaires sont régulièrement relevés, ce sont surtout les impacts environnementaux auxquels il est le plus souvent fait référence (Coccossis, 2001). Le développement touristique, pensons seulement aux complexes hôteliers en bord de mer, a effectivement déjà dégradé de nombreux écosystèmes. Dans les régions côtières et montagneuses, connues pour leur vulnérabilité, nombre d'impacts environnementaux ont été observés : atteinte à l'intégrité physique du territoire et des paysages, exploitation des ressources, coûts énergétiques des transports, pollution au sens large, impacts sur la biodiversité (Behnassi, 2008 : 7; Grenier, 2000), phénomènes directement proportionnels à la croissance mondiale du tourisme (Cazelais et al., 1999; Paul et al., 2007).

Ce qui vaut pour le tourisme en général trouve également à s'appliquer à l'écotourisme. Cette forme de tourisme peut être définie comme "environmentally responsible travel and visitation to relatively undisturbed natural areas, in order to enjoy and appreciate nature (and any accompanying cultural features - both past and present) that promotes conservation, has low negative visitor impact, and provides for beneficially active socioeconomic involvement of local populations» (Ceballos-Lascuráin, 1993 : 20). Or, malgré son objectif de conservation de la nature, l'activité écotouristique serait génératrice d'impacts environnementaux croissants non négligeables (AEQ, 2004; KPMG, 2010). L'écotourisme n'échappe par ailleurs pas à la double dynamique de contemplation/conservation de la nature et du développement du territoire et de ses communautés. Dans ce contexte, la difficulté particulière est que toute atteinte aux capacités de seuil et à la régénération des écosystèmes mine par le fait même la spécificité et la raison d'être de l'écotourisme. Un projet écotouristique se distingue en effet par la forte valeur écologique du territoire de référence. Dit autrement, le substrat environnemental peut être considéré comme un facteur prioritaire et limitatif de l'écotourisme (Boutaud, 2005 : 73). C'est ce qui nous amène à poser la question du comment ou de l'opérationnalisation des projets écotouristiques dans une perspective de développement durable des territoires.

Pour ce, l'approche évaluative des projets, identifiant des critères discriminants du point de vue des promoteurs et des gestionnaires, a été privilégiée. Celle-ci permet de répondre à la question suivante : lors de la phase de conception d'un projet écotouristique, destiné à mettre en valeur les ressources naturelles d'un territoire, donc à le développer, quels paramètres les promoteurs du projet devraient-ils prioritairement considérer pour favoriser son développement durable? Le premier objectif de cet article est donc d'identifier les critères de la durabilité écotouristique, ici définie comme l'application et l'adaptation des principes environnementaux, économiques et sociaux du développement durable aux spécificités de l'écotourisme. Le second est d'analyser plus précisément les critères pouvant mener à la durabilité environnementale in situ. Car si cette dernière ne constitue pas l'unique critère de la durabilité écotouristique, elle en est le substrat.

Cette analyse sera conduite au regard des théories de la science régionale et du développement territorial et selon une approche opérationnelle. Le développement régional et territorial explore les dimensions économique, sociale, historique et géographique du développement en mettant à contribution les différentes sciences humaines et sociales. Un axe de réflexion est notamment la question de l'aménagement et de la gestion durables des territoires et des ressources, ainsi que les nouveaux rapports entretenus par les sociétés contemporaines avec la nature et leur environnement. Ces nouveaux rapports peuvent remettre en question les manières d'occuper et d'habiter les territoires, et façonner de nouveaux modèles de développement, notamment durables.

\section{La méthodologie}

Nous définissons les critères de la durabilité écotouristique comme ceux qui favorisent l'ancrage de l'écotourisme dans le développement durable des territoires concernés. Il s'agit de balises qui permettent de bâtir des liens opérationnels entre cette forme de tourisme de nature et le développement durable des territoires. Ces critères contribuent à l'intégration à la fois des composantes ou piliers du développement durable, des temps et des acteurs du développement territorial (Gagnon, 2012). Pour faciliter leur opérationnalisation, une identification précise est requise. Pour y parvenir, nous retenons une méthodologie en deux étapes. La première étape vise à caractériser et discriminer les critères de la durabilité écotouristique. Il s'agit de déterminer à partir de quelles sources définitionnelles ces critères peuvent être distingués et décrits. Une fois ce cadre établi, la seconde étape vise à obtenir un relevé de ces critères et de leurs composantes, à les identifier individuellement et à les mettre en perspective pour s'assurer de leur exhaustivité et pour faciliter par la suite leur mise en œuvre.

Pour caractériser les critères de la durabilité écotouristique, la méthodologie adoptée repose sur une analyse croisée de définitions sur l'écotourisme, le développement durable et le tourisme durable. Dans les faits, ce sont en effet surtout et traditionnellement les critères du tourisme durable qui sont inventoriés, l'écotourisme n'étant que rarement ciblé directement. Nous postulons alors qu'un croisement entre les littératures sur l'écotourisme et le tourisme durable permettra la caractérisation des critères de la durabilité écotouristique. L'analyse de l'existence des liens théoriques entre ces concepts a été réalisée à partir de sources institutionnelles spécialisées.

L'étape suivante, l'identification des critères de la durabilité écotouristique, à partir de critères de l'écotourisme et du tourisme durable, a fait l'objet d'une méta-analyse basée sur 125 textes de référence essentiellement francophones, issus de deux catégories :

a) des textes officiels provenant d'organismes collectifs publics ou privés spécialisés (Ce corpus est essentiellement composé de rapports des principales institutions mondiales, nationales et provinciales et des programmes européens de 
développement touristique chargés de la détermination et de la mise en ouvre des critères du tourisme durable et de l'écotourisme. Par exemple : OMT, AFIT, MDDEP);

b) des articles et recherches scientifiques, rédigés majoritairement par des chercheurs universitaires spécialisés, et portant soit sur le durable tourisme, soit sur l'écotourisme (ex : Gagnon, Breton, Blamey, Céron et Dubois).

\section{La caractérisation des critères de la durabilité écotouristique}

À partir de cinq textes institutionnels de référence (PNUE, 1995; OMT, 2005; Québec, 2005; PNUE et OMT, 2007; Comité des Régions, 2011), nous avons constaté qu'il existe un socle commun comportant sept éléments définitionnels du tourisme durable (voir tableau 1).

Pour parvenir à ce constat, les éléments définitionnels énoncés dans ces textes ont été comparés entre eux, et ceux ayant suffisamment de similarités ont été amalgamés. Les éléments qui apparaissent de manière isolée ont été conservés, à l'exception de la «satisfaction des visiteurs», issue de la Charte du tourisme durable (PNUE, 1995), qui nous apparaît comme non discriminante du point de vue de notre étude. Ces éléments définitionnels du tourisme durable sont les suivants :

- l'intégrité environnementale : préservation de la diversité et des processus biophysiques essentiels;

- l'utilisation rationnelle et intégrée des ressources : conciliation du développement avec les besoins et les capacités du territoire;

- la sensibilisation accrue des exploitants et des touristes à

l'utilisation durable de l'environnement;

- la participation des acteurs locaux;

- le respect de l'authenticité socioculturelle des communautés hôtes;
- le bien-être et les bénéfices socio-économiques équitables pour les communautés locales;

- la viabilité et la prospérité économiques.

Ces sept éléments définitionnels recoupent ceux retenus par l'Organisation mondiale du tourisme (PNUE et OMT, 2007). L'OMT (2005) souligne que le tourisme durable n'est donc pas une forme distincte ou spécifique de tourisme. Elle précise que «[1]es principes directeurs du développement durable et les pratiques de gestion durable du tourisme sont applicables à toutes les formes de tourisme dans tous les types de destination y compris au tourisme de masse et aux divers créneaux touristiques» (OMT, $2005: 1)$.

Les définitions de l'écotourisme ont elles aussi été relevées à partir de sources spécialisées (voir tableau 2). À ces définitions a été ajouté le relevé des "métaprincipes» de l'écotourisme, issus de la recherche de Gagnon et Lapointe (2006), la détermination de ceux-ci procédant elle-même d'une analyse d'un corpus de 25 textes fondateurs.

Cinq éléments définitionnels de l'écotourisme ont pu être identifiés :

- l'observation et l'interprétation du milieu naturel et du patrimoine culturel traditionnel;

- la valorisation (éducation, responsabilisation) de la conservation et de l'éthique de l'environnement;

- la génération d'une expérience touristique authentique et personnalisée;

- la participation des communautés locales dans la planification, le développement et l'exploitation;

- la contribution équitable au bien-être socio-économique des communautés locales.

Aux quatre «métaprincipes» de l'écotourisme de Gagnon et Lapointe (2006) s'ajouteraient donc essentiellement l'observation et l'interprétation du milieu naturel et du

Tableau 1: Comparaison des éléments définitionnels du tourisme durable à partir de sources institutionnelles spécialisées

\begin{tabular}{|c|c|c|c|c|c|}
\hline SOURCES & $\begin{array}{l}\text { CHARTE DU TOURISME } \\
\text { DURABLE } \\
\text { (PNUE, 1995) }\end{array}$ & $\begin{array}{l}\text { ORGANISATION } \\
\text { MONDIALE DU TOURISME } \\
\text { (OMT, 2005) }\end{array}$ & $\begin{array}{l}\text { VERS UN TOURISME } \\
\text { DURABLE. POLITIQUE } \\
\text { TOURISTIQUE DU QUÉBEC } \\
\text { (QUÉBEC, 2005) }\end{array}$ & $\begin{array}{l}\text { COMITÉ FRANÇAIS POUR } \\
\text { LE DÉVELOPPEMENT DU } \\
\text { TOURISME DURABLE } \\
\text { (PNUE ET OMT, 2007) }\end{array}$ & $\begin{array}{l}\text { PROJET DE CADRE } \\
\text { POLITIQUE POUR LE } \\
\text { TOURISME EUROPÉEN } \\
\text { (COMITÉ DES RÉGIONS, 2011) }\end{array}$ \\
\hline $\begin{array}{l}\text { Éléments } \\
\text { définitionnels }\end{array}$ & $\begin{array}{l}\text { - viabilité économique; } \\
\text { - prospérité au niveau local; } \\
\text { - qualité de l'emploi; } \\
\text { - équité sociale; } \\
\text { - satisfaction des visiteurs; } \\
\text { - contrôle local; } \\
\text { - bien-être des communautés; } \\
\text { - richesse culturelle; } \\
\text { - intégrité physique; } \\
\text { - diversité biologique; } \\
\text { - utilisation rationnelle } \\
\text { des ressources; } \\
\text { - pureté de l'environnement }\end{array}$ & $\begin{array}{l}\text { - usage optimal des ressources } \\
\text { environnementales; } \\
\text { - respect de l'authenticité } \\
\text { socioculturelle } \\
\text { des communautés; } \\
\text { - offre d'avantages socio- } \\
\text { économiques pour tous } \\
\text { les intervenants }\end{array}$ & $\begin{array}{l}\text { - viabilité économique } \\
\text { des régions; } \\
\text { - rentabilité des entreprises } \\
\text { touristiques; } \\
\text { - sensibilisation accrue } \\
\text { des exploitants et de touristes } \\
\text { à l'utilisation durable } \\
\text { de l'environnement; } \\
\text { - offre touristique développée } \\
\text { et exploitée dans le respect } \\
\text { des populations locales }\end{array}$ & $\begin{array}{l}\text { - articulation des modes } \\
\text { de production et de } \\
\text { consommation responsables; } \\
\text { - offre d'avantages socio- } \\
\text { économiques équitablement } \\
\text { répartis aux populations } \\
\text { qui vivent, travaillent ou } \\
\text { séjournent sur cet espace; } \\
\text { - aménagement et gestion } \\
\text { intégrés des ressources; } \\
\text { - participation des acteurs } \\
\text { locaux; } \\
\text { - conciliation } \\
\text { du développement avec } \\
\text { les besoins et les capacités } \\
\text { du territoire }\end{array}$ & $\begin{array}{l}\text { - contribution économique au } \\
\text { développement des futures } \\
\text { générations (équité et } \\
\text { efficacité); } \\
\text { - compatibilité avec la culture, } \\
\text { les valeurs et l'identité des } \\
\text { régions européennes; } \\
\text { - compatibilité avec la } \\
\text { préservation des processus } \\
\text { environnementaux essentiels; } \\
\text { - préservation de la diversité } \\
\text { et des ressources biologiques }\end{array}$ \\
\hline
\end{tabular}

Source : compilation des auteurs. 
patrimoine culturel traditionnel. L'intégration des principes de développement durable comme moyen pour en favoriser l'opérationnalisation apparaît également de manière transversale. Ces éléments définitionnels sont suffisamment discriminants dans la littérature pour être retenus.

\section{Analyse des convergences et spécificités}

L'observation d'une superposition des éléments définitionnels de l'écotourisme et du tourisme durable conduit à définir des critères de la durabilité écotouristique à partir de leurs éléments définitionnels communs et distinctifs.

Selon notre interprétation, l'utilisation rationnelle et intégrée des ressources et l'éducation relative à l'environnement, l'équité et le bien-être socio-économique des communautés locales, ainsi que la participation des acteurs locaux s'avèrent des éléments communs au tourisme durable et à l'écotourisme. Plus globalement, les résultats de cette analyse comparative soulignent de manière assez patente une correspondance entre les éléments définitionnels issus des deux concepts et pratiques. Nous relevons, comme l'International Ecotourism Society le souligne elle-même (1990), que l'écotourisme est une forme de tourisme durable, et qu'il l'est même de manière consubstantielle (Salaméro, 2010; Villeneuve, s. d). Il fait appel aux principes de développement durable (Desmarais, 2007) et il peut être une référence utile dans le cadre de stratégies de tourisme plus durable (OMT, 2005 ; CCE, 2000).

Selon Gagnon et Lapointe (2006 : 21), l'écotourisme s'inscrit dans une approche environnementaliste, une des quatre approches du développement durable. Selon cette approche, un projet touristique serait durable lorsqu' il permet aux écosystèmes et aux ressources qui y sont rattachées de rester disponibles pour répondre à une fonction économique. À contrario, si un projet ne contribue pas à améliorer les écosystèmes, c'est le flux des produits et des services qui se tarit, entraînant son lot de conséquences sociales. L'écotourisme vise donc la mise en œuvre des principes du développement durable. L'écotourisme tend vers ce dernier. Il n'en est pas la réalisation à priori.

Le tourisme durable, en revanche, a vocation à s'appliquer à toute forme de tourisme. Rappelons que l'extension du concept de développement durable au tourisme a pris forme lors de la Conférence mondiale du tourisme durable à Lanzarote en 1995. Cette conférence a donné lieu à la publication de la Charte du tourisme durable qui s'inspire des principes énoncés dans la Déclaration de Rio sur l'environnement et le développement et des recommandations de l'Agenda 21. Le tourisme durable y est abordé comme un cadre générique de pratique du tourisme. Il correspond à «toute forme de développement, d'aménagement ou activité touristique qui respecte et préserve à long terme les ressources naturelles, culturelles et sociales, et contribue de manière positive et équitable au développement économique et à l'épanouissement des individus qui vivent, travaillent et séjournent dans ces espaces» (AFIT, cité par Breton, $2006: 60$ ).

L'écotourisme n'est pas pour autant un synonyme de tourisme durable, car s'il adhère bel et bien à certains éléments définitionnels, il comporte aussi des éléments distinctifs. L'écotourisme fait en ce sens explicitement référence à un produit bien spécifique. L'observation et l'interprétation du

Tableau 2: Comparaison des éléments définitionnels de l'écotourisme

\begin{tabular}{|c|c|c|c|c|c|c|}
\hline SOURCES & $\begin{array}{l}\text { GAGNON ET } \\
\text { LAPOINTE, } 2006\end{array}$ & $\begin{array}{l}\text { NORME P-9700-060, } \\
\text { TOURISME - PRODU- } \\
\text { IT D'ÉCOTOURISME } \\
\text { (BNQ, 2003) }\end{array}$ & $\begin{array}{l}\text { ECOTOURISME } \\
\text { FRANCE }\end{array}$ & $\begin{array}{l}\text { AGENCE POUR LE } \\
\text { DÉVELOPPEMENT } \\
\text { DE L'ECOTOURISME } \\
\text { (ADE) }\end{array}$ & $\begin{array}{l}\text { CEBALLOS-LASC- } \\
\text { URAIN (UICN), } 1996\end{array}$ & $\begin{array}{l}\text { DÉCLARATION } \\
\text { DE QUÉBEC SUR } \\
\text { L'ÉCOTOURISME }\end{array}$ \\
\hline $\begin{array}{l}\text { Éléments } \\
\text { définitionnels }\end{array}$ & $\begin{array}{l}\text { - valorisation } \\
\text { de la conservation } \\
\text { de l'environnement; } \\
\text { - contribution équitable } \\
\text { au développement } \\
\text { économique; } \\
\text { - prise en compte } \\
\text { et réponse aux besoins } \\
\text { des communautés } \\
\text { hôtes; } \\
\text { - génération d'une } \\
\text { expérience touristique } \\
\text { nouvelle, authentique } \\
\text { et responsable }\end{array}$ & $\begin{array}{l}\text { - valorisation et } \\
\text { préservation du milieu } \\
\text { naturel; } \\
\text { - interprétation des } \\
\text { composantes naturelles } \\
\text { ou culturelles du milieu; } \\
\text { - repose sur des notions } \\
\text { de développement } \\
\text { durable; } \\
\text { - bénéfices socio- } \\
\text { économiques pour les } \\
\text { communautés locales } \\
\text { et régionales }\end{array}$ & $\begin{array}{l}\text { - objectif d'observation } \\
\text { de la nature et des } \\
\text { cultures traditionnelles; } \\
\text { - éducation } \\
\text { et interprétation } \\
\text { de l'environnement; } \\
\text { - organisé pour des } \\
\text { groupes restreints par } \\
\text { de petites entreprises } \\
\text { locales spécialisées; } \\
\text { - protection des zones } \\
\text { naturelles; } \\
\text { - avantages socio- } \\
\text { économiques pour } \\
\text { les communautés } \\
\text { d'accueil; } \\
\text { - promotion de la } \\
\text { nécessité de préserver } \\
\text { le capital naturel et } \\
\text { culturel }\end{array}$ & $\begin{array}{l}\text { - conservation des sites } \\
\text { d'intérêt écologique } \\
\text { et culturel; } \\
\text { - sensibilisation, } \\
\text { éducation et formation } \\
\text { de toutes les parties } \\
\text { prenantes concernées; } \\
\text { - promotion de pratiques } \\
\text { responsables et } \\
\text { de comportements } \\
\text { éthiques; } \\
\text { - épanouissement } \\
\text { économique, social, } \\
\text { culturel des populations } \\
\text { hôtes; } \\
\text { - préservation } \\
\text { et valorisation } \\
\text { du patrimoine naturel } \\
\text { et sociétal }\end{array}$ & $\begin{array}{l}\text { - responsabilité } \\
\text { environnementale; } \\
\text { - préservation du milieu } \\
\text { naturel ainsi que toute } \\
\text { manifestation culturelle } \\
\text { passée ou présente } \\
\text { observable depuis ces } \\
\text { milieux; } \\
\text { - promotion de la } \\
\text { conservation; } \\
\text { - participation active } \\
\text { des populations locales } \\
\text { dans le but de générer } \\
\text { des avantages }\end{array}$ & $\begin{array}{l}\text { - contribution à la } \\
\text { protection du patrimoine } \\
\text { naturel et culturel; } \\
\text { - inclusion des } \\
\text { communautés locales } \\
\text { dans la planification, } \\
\text { le développement et } \\
\text { l'exploitation; } \\
\text { - contribution au bien- } \\
\text { être des communautés } \\
\text { locales; } \\
\text { - interprétation } \\
\text { du patrimoine naturel } \\
\text { et culturel; } \\
\text { - voyage en individuel } \\
\text { pour de petits groupes }\end{array}$ \\
\hline
\end{tabular}

Source : compilation des auteurs. 


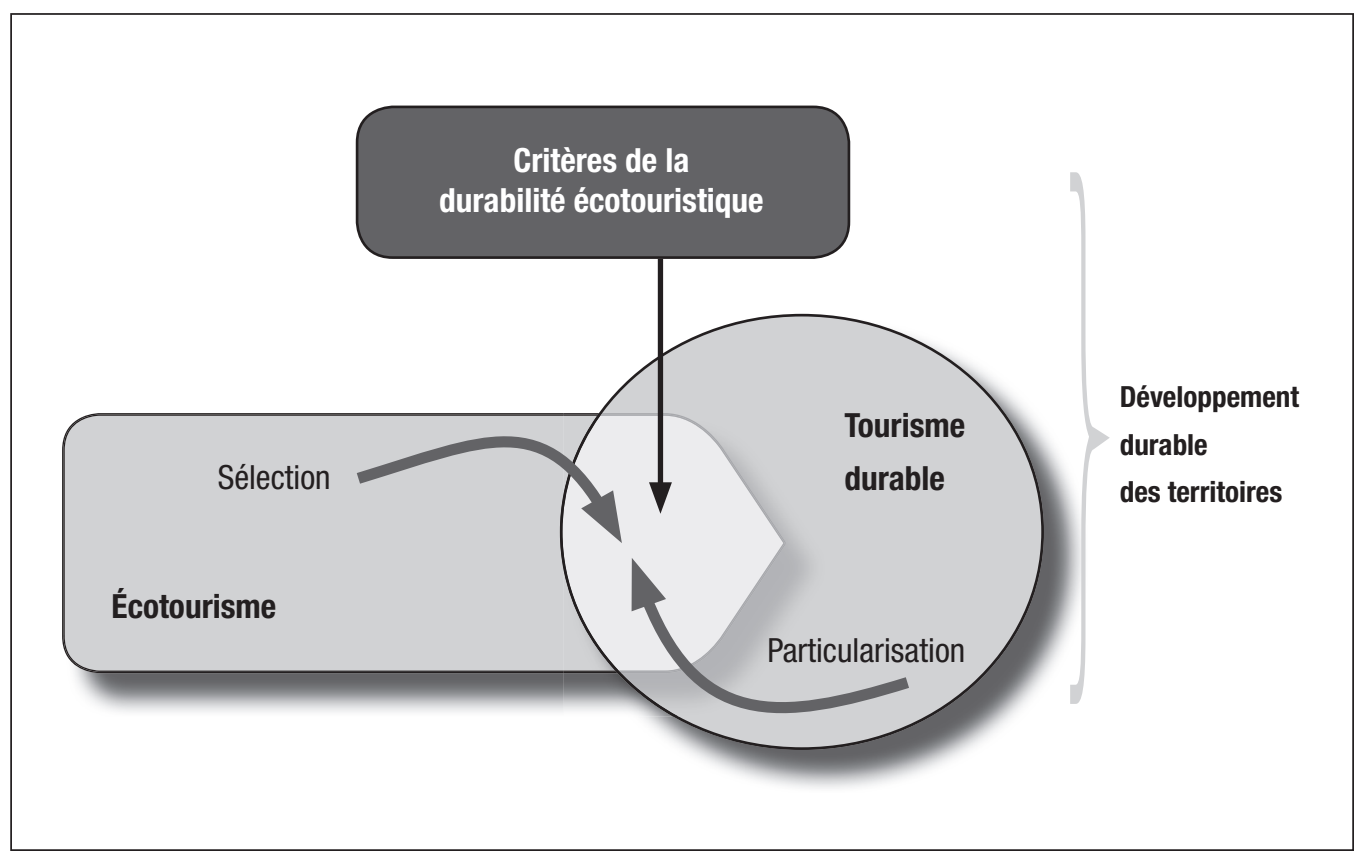

ILLUSTRATION 1 : Caractérisation des critères de la durabilité écotouristique

(source : compilations des auteurs). milieu naturel et du patrimoine culturel traditionnel ou la génération d'une expérience touristique authentique et personnalisée le distinguent notamment.

L'écotourisme est ainsi avant tout un tourisme de nature. Le tourisme de nature (nature-based tourism, en anglais) est une forme de tourisme à laquelle on peut associer l'ensemble des produits et activités touristiques utilisant l'environnement naturel pour se réaliser, se concrétiser (Couture, 2002 : 9). Il correspond à "any type of tourism that relies on attractions directly related to the natural environment» (Weaver, 2001 : 16). Les activités du tourisme de nature sont axées sur l'observation ou l'appréciation du milieu naturel à des fins de découverte ou de pratique d'activités de plein air. Elles induisent une démarche participative des clientèles, une implication sensible et émotionnelle, une participation plutôt qu'une consommation. Il s'agit pour les clients de vivre une expérience, d'être acteurs de leur découverte (Macouin et Pierre, 2003 : 30 ; Breton, 2006).

Toutes les formes de tourisme de nature ne sont cependant pas compatibles avec l'écotourisme : certaines incluent du prélèvement faunique - tourisme cynégétique et halieutique —, d'autres des modes de transport motorisés — expédition de motoneige. D'autres encore utilisent le milieu naturel comme cadre d'activités sans que la découverte de ce milieu soit l'objet précis du séjour : les activités pratiquées dans une perspective sportive - le golf, le ski alpin, etc. — et le camping pris comme mode d'hébergement ne sont pas considérés comme des pratiques écotouristiques.

L'écotourisme se distingue en ce qu'il est le seul à valoriser explicitement la sensibilisation environnementale - volet éducatif - et le bien-être de la population hôte (Desmarais, 2007; Tardif, 2003). Il s'agit d'un tourisme dynamique où la nature n'est pas simplement objet de contemplation. Dans une logique proactive, les activités et les pratiques sont conçues pour favoriser la conservation du milieu (Sommet mondial de l'écotourisme, 2002 : 70). Les aménités et les attraits environnementaux du lieu fondent la valeur de l'expérience écotouristique. À ce titre, la richesse de l'environnement et de la biodiversité s'avère un métaprincipe inéluctable. Ainsi Denais (2007 : 41) estime que «[1]a valorisation de l'environnement est le "métaprincipe" le plus familier de l'écotourisme. Il se distingue ainsi du tourisme conventionnel ou tourisme de masse du point de vue des impacts environnementaux. En effet, c'est le premier principe que l'on retrouve dans la grande majorité des définitions de l'écotourisme qui mettent l'accent sur la préservation de l'environnement grâce à cette activité».

Globalement, aucune restriction n'existe quant au lieu de pratique du tourisme durable — comme c'est le cas pour l'écotourisme - , et l'écotourisme n'est pas l'expression singulière du développement durable dans la sphère touristique. Plus spécifiquement, l'écotourisme ajoute aux éléments définitionnels du tourisme durable des critères distinctifs. L'existence d'activités d'interprétation des composantes naturelles du milieu dans le cadre d'expériences touristique authentiques et personnalisées en est un exemple issu de la littérature sélectionnée. À contrario, toutes les formes de tourisme durable ne se pratiquent pas nécessairement dans des régions naturelles et ne sont pas précisément organisées pour de petits groupes, par exemple.

En conséquence, tous les éléments définitionnels de l'écotourisme identifiés dans le tableau 2 ne sont pas propres au développement durable. Tous les critères du tourisme durable ne sont pas spécifiquement paramétrés pour l'écotourisme. C'est en comparant les définitions des deux tableaux précédents que nous estimons que la caractérisation des critères de la durabilité écotouristique passe par un raffinement des critères de l'écotourisme et de ceux du tourisme durable. 


\section{Raffinement des critères de la durabilité écotouristique}

Ce raffinement pourrait être opéré selon deux modalités. La première conduirait à une sélection, parmi les critères de l'écotourisme, de ceux qui sont précisément inscrits dans une démarche territoriale de développement durable. La seconde nécessiterait une particularisation des critères du tourisme durable pour n'en retenir que ceux qui sont adaptés à l'écotourisme, notamment en ce qui a trait à sa dimension environnementale, c'est-à-dire à son statut de tourisme de nature et à la criticité de la gestion de l'environnement. L'illustration 1 représente la double démarche permettant la caractérisation des critères de la durabilité écotouristique comme préalable à leur identification.

\section{L'identification et la hiérarchisation des critères de la durabilité écotouristique}

Suite à une étude de sources secondaires de la durabilité écotouristique identifiées ci-après, seront tour à tour présentés les résultats bruts puis les analyses conclusives que ces derniers ont générées, notamment au regard de la hiérarchisation des critères de la durabilité écotouristique. Cette hiérarchisation constitue un résultat d'analyse puisqu'elle permet, pour la première fois, de classifier ces critères selon l'importance qui peut leur être attribuée au regard de leur fréquence dans les documents officiels et dans la littérature scientifique.

Pour parvenir à une identification des critères de la durabilité écotouristique, une base de données a été constituée à partir de 125 textes de référence essentiellement francophones : des textes officiels provenant d'organismes collectifs publics ou privés spécialisés et des articles et recherches scientifiques, rédigés majoritairement par des chercheurs universitaires spécialisés. À partir de ces documents, des critères à prendre en compte pour favoriser le développement territorial durable d'un projet écotouristique ont été identifiés et regroupés au sein d'une grille, selon leur similitude ou parenté thématique. Pour rendre l'identification des critères plus complète et précise, cette grille a été enrichie de données techniques. Des études et rapports techniques issus de cabinets de consultants spécialisés ou d'entrepreneurs touristiques et portant sur l'analyse de cas de mise en œuvre de projets écotouristiques ont ainsi été analysés. L’objectif était ici de relever des paramètres que les professionnels sont invités à utiliser pour faciliter la durabilité de leurs projets et qui ont pu être omis dans les écrits à caractère institutionnel et scientifique. Les raisons de ces éventuelles omissions peuvent être variées. Les institutions internationales ou nationales en charge du développement touristique durable sont animées par des logiques diverses qui peuvent les amener à considérer comme plus ou moins importantes certaines composantes de l'écotourisme. Des aspects comme le développement et le progrès humains peuvent ainsi prendre le pas sur des considérations plus spécifiques et plus directement exploitables par les promoteurs. Par ailleurs, les spécificités de l'écotourisme peuvent parfois se perdre et se diluer dans des réflexions générales sur le tourisme. De la même manière, certains auteurs scientifiques abordent la question de la durabilité selon une approche théorique très spécifique qui tend parfois à « déconnecter» leurs conclusions des réalités et préoccupations du terrain. Mettre en place un système de traçabilité des actions ou encore développer un plan de gestion des risques sont des exemples de critères issus des rapports techniques.

Si chacune des catégories de sources, prises isolément, ne livre qu'une partie des critères, leur cumul permet toutefois la bonification de la grille initiale et un relevé plus détaillé. La démarche de comparaison systématique vise à procéder à des synthèses et des regroupements de données par le biais de la méta-analyse. Cette méthode consiste dans l'analyse simultanée d'un ensemble d'études s'intéressant à la même question, dans le but d'obtenir des informations qu'aucune de ces études prises isolément ne pourrait fournir. L'objectif était d'obtenir un résultat global agrégatif, le nombre, la diversité et le croisement des sources apportant une certaine validité aux résultats de la recherche.

\section{Les critères génériques}

Le premier résultat tangible de cette recherche est l'élaboration d'une grille des critères contributifs au développement durable des projets écotouristiques. La grille produite est constituée de 35 critères génériques ou d'interprétation large. Ces critères se déclinent ensuite en 130 critères spécifiques qui précisent et détaillent les premiers. Ces critères concernent chacun des trois volets du développement durable, soit l'économie, l'environnement et le social. La gouvernance, comprise généralement comme la démocratisation des processus de choix, jugée parfois comme un quatrième critère du développement durable (Brodhag, 1999; Gagnon, 2008), a été ici incluse dans le volet social. L'approche extensive qui en fait un volet à part entière reste en effet encore marginale dans les textes étudiés.

Les critères génériques identifiés dans la littérature - et présentés dans le tableau 3 - sont relativement nombreux et s'inscrivent de manière évidente dans la philosophie du développement durable dont ils empruntent la terminologie (équité, gouvernance, etc.). Certains critères évoquent plus distinctement une application ciblée au secteur écotouristique (valorisation de l'identité et du patrimoine local, gestion des interactions visiteurs-biodiversité). Les critères évoquant des processus techniques (innovation, mesures de protection du milieu, démarche qualité, etc.) sont moins cités que les critères les plus génériques. L'ensemble constitue cependant un corpus assez homogène, sans véritable critère prépondérant. La grille permet de faire notamment état d'un certain équilibre des critères selon les piliers du développement durable qui apparaissent comme équipollents au regard de leur nombre respectif.

Cet équilibre des critères selon les piliers du développement durable illustre toutefois le fait que si les premières définitions de l'écotourisme mettaient l'emphase sur une proximité recherchée avec la nature par les touristes (Ceballos-Lascuráin, 1987), les définitions plus récentes (Honey, 1999; Blamey, 2001) ont plutôt cherché à mettre en lumière une variété de principes associés au concept de développement durable. Bien plus, le centre de gravité de l'écotourisme semblerait désormais se déplacer autour de considérants sociaux. En l'espèce, la présence des critères «Bien-être des communautés d'accueil» et "Gouvernance participative», dont l'analyse quantitative 
Tableau 3: Critères génériques de la durabilité écotouristique

\begin{tabular}{|c|c|c|}
\hline & Volet du développement durable & Critères \\
\hline 1. & Environnemental & Respect des obligations légales en matière environnementale \\
\hline 2. & Environnemental & Utilisation d'outils d'évaluation et de contrôle spécialisés \\
\hline 3. & Environnemental & Éducation relative à l'environnement \\
\hline 4. & Environnemental & Préservation de l'intégrité géophysique du milieu (air, sol, eau) \\
\hline 5. & Environnemental & Préservation de l'intégrité patrimoniale, de l'identité du site et des paysages \\
\hline 6. & Environnemental & Préservation de l'intégrité écologique du site (biodiversité, écosystèmes) \\
\hline 7. & Environnemental & Maintien de la pureté de l'environnement \\
\hline 8. & Environnemental & Utilisation rationnelle des ressources \\
\hline 9. & Environnemental & Contribution proactive à la protection du milieu \\
\hline 10. & Environnemental & Gestion des interactions visiteurs-biodiversité (faune, flore) \\
\hline 11. & Environnemental & Mise en valeur du patrimoine naturel \\
\hline 12. & Économique & Maîtrise des outils et mécanismes de gestion \\
\hline 13. & Économique & Rentabilité financière des projets écotouristiques \\
\hline 14. & Économique & Planification et stratégie de développement \\
\hline 15. & Économique & Offre écotouristique ciblée et adaptée \\
\hline 16. & Économique & Démarche qualité transversale \\
\hline 17. & Économique & Contrôle et suivi des activités/projets \\
\hline 18. & Économique & Marketing efficace et responsable \\
\hline 19. & Économique & Communication efficace et éthique \\
\hline 20. & Économique & Valorisation des emplois \\
\hline 21. & Économique & Promotion de la formation de la main-d'œuvre \\
\hline 22. & Économique & Coordination des agents économiques \\
\hline 23. & Économique & Implication des acteurs locaux \\
\hline 24. & Économique & Entrepreneuriat proactif et innovation \\
\hline 25. & Social & Adhésion du milieu d'accueil aux projets touristiques \\
\hline 26. & Social & Connectivité civile et cohésion sociale \\
\hline 27. & Social & Respect des droits et structures sociales des communautés autochtones et traditionnelles \\
\hline 28. & Social & Respect de la communauté d'accueil par le promoteur \\
\hline 29. & Social & Sécurité des visiteurs \\
\hline 30. & Social & Bien-être des communautés d'accueil \\
\hline 31. & Social & Bien-être des visiteurs \\
\hline 32. & Social & Valorisation de l'identité et du patrimoine culturel de la communauté d'accueil \\
\hline 33. & Social & Gouvernance participative \\
\hline 34. & Social & Inclusion des projets touristiques dans une stratégie de développent local \\
\hline 35. & Social & Répartition large et équitable des bénéfices socio-économiques \\
\hline
\end{tabular}

souligne qu'ils occupent les deux premiers rangs en termes d'occurrence, illustre ce point. Preuve en est également que, parmi les recherches récentes portant sur l'organisation durable de l'industrie écotouristique, la question de la gouvernance des populations hôtes semble au cœur des débats. Pour certains auteurs (Gagnon, 2008; Lequin, 2000), c'est en effet autour des processus de consultation, de participation et de contribution de la communauté d'accueil au processus décisionnel que semble reposer la construction d'un modèle optimal, durable, de gestion de l'industrie.
Cette interprétation de la durabilité écotouristique illustre une tendance selon laquelle, «avec l'évolution des pratiques alternatives et responsables, il semble que ce concept [l'écotourisme] a quelque peu délaissé la base même, qui est le milieu naturel, pour se concentrer essentiellement sur le développement durable» (Laliberté, $2011: 1$ ). Cette tendance conduit à un certain renversement conceptuel de la primauté environnementale au bénéfice du volet social comme socle de l'écotourisme. Elle serait cependant selon nous plus symptomatique d'une posture d'analyse que 
d'une réalité de terrain où les ressources naturelles demeurent des composantes critiques et prioritaires. Cette lecture idéologique remet en cause la primauté du maintien de la disponibilité des ressources naturelles comme condition première et consubstantielle de l'écotourisme, telle que pourtant relevée par certains auteurs (Boutaud, 2005; Dowling, 2006; Denais, 2007; de Juan Alonso, 2010; Tranquard, 2013). C'est en convenant de cette primauté que nous présentons, dans le cadre de la présente analyse, le relevé des critères spécifiques de la durabilité environnementale.

\section{Les critères de la durabilité environnementale}

Le tableau 3 ne rend pas compte de la totalité des résultats de la démarche de recherche entreprise puisqu'il ne mentionne pas les 130 critères spécifiques qui se trouvent à caractériser et préciser les critères génériques. Suivant une approche «traditionnelle» ou «définitionnelle» de l'écotourisme qui entérine une importance prioritaire de la durabilité environnementale comme condition d'opérationnalisation, nous proposons cependant ici le relevé des critères spécifiques du volet environnemental (voir tableau 4).

Une analyse de ces critères spécifiques permet de noter leur dimension intégrative puisque bon nombre d'entre eux peuvent s'inscrire dans un continuum, un processus stratégique d'implantation des principes du développement durable - évaluation puis respect de la capacité de charge, réduction de la consommation puis gestion des déchets, etc. Les critères spécifiques invitent par ailleurs à des démarches proactives de la part des gestionnaires où les principes de précaution recours systématique à des évaluations environnementales et à des études d'impact —, de traçabilité des actions et de respect des composantes du site d'activité - patrimoine, capital naturel et culturel, paysage et typicité des infrastructures devraient être assurés.

En considérant les modalités de mise en œuvre de ces critères, des outils techniques semblent poindre au travers du relevé effectué. Sans être directement nommés, on perçoit qu'un certain nombre d'outils pourraient supporter efficacement une démarche d'opérationnalisation du développement durable. Par exemple :

- des outils scientifiques pour l'évaluation des impacts;

- des outils juridiques pour les démarches de certification et de contrôle;

- des outils pédagogiques pour l'éducation relative à l'environnement;

- des outils de communication pour la diffusion et le partage d'informations.

Soulignons par ailleurs qu'en précisant les critères à mettre en œuvre, la grille obtenue se rapproche elle-même d'un outil d'aide à la mise en place concrète de la durabilité écotouristique.

\section{La pondération et la territorialisation des critères}

Les modalités méthodologiques retenues dans le cadre de cette recherche pour identifier les critères de la durabilité écotouristique pourraient favoriser leur opérationnalisation. Tous les critères relevés ont, en effet, été analysés selon la fréquence de leurs occurrences dans les sources étudiées, soit les résultats d'une analyse quantitative basée sur plus de 2200 références croisées. Une hiérarchisation des critères a ainsi pu être obtenue, chaque occurrence augmentant leur pondération (voir tableau 5). Par exemple, le critère du «Bienêtre des communautés d'accueil » a été le plus cité parmi l'ensemble des sources en tant que critère favorisant la durabilité des projets touristiques et écotouristiques ( 70 occurrences au total), ce qui affirme sa dimension sociale. Toutefois, la pondération des critères est limitée à la fréquence de leurs occurrences, soit le nombre de fois où ils sont précisément cités comme des facteurs du développement durable en contexte écotouristique. Elle n'est pas liée à la valeur intrinsèque que les divers auteurs des études consultées leur ont attribuée. La récurrence de leurs citations dans les textes sources pourrait cependant constituer un indice de la pertinence des critères ou à tout le moins faciliter leur mise en perspective dans l'objectif de prioriser des critères importants lors de l'opérationnalisation des projets.

L'analyse conduite permet également de souligner que certains critères de la durabilité écotouristique identifiés convergent vers une lecture territoriale de la durabilité écotouristique en abordant la conservation de l'identité et la dynamique du territoire et de ses composantes, soit population, ressources, patrimoine : "Bien-être», «Gouvernance participative» et «Préservation de l'intégrité géophysique du milieu (air, sol, eau)», mais aussi «Utilisation rationnelle des ressources» et «Valorisation de l'identité et du patrimoine culturel de la communauté d'accueil». Il s'agit d'un autre élément à considérer lors d'une démarche de conception de produits écotouristiques durables.

\section{Conclusion}

Au cours des dernières années, le tourisme durable a fait l'objet d'une large promotion d'esprit normatif (OMT, 2005; Desvignes, 2000; AFIT, 2001). Des interrogations demeurent cependant quant à ses bases théoriques (Lauriol, 2004) ou à sa capacité opérationnelle (Ceron et Dubois, 2002; Ryan, 2002; Marsat, 2008). La mise en œuvre de la durabilité se heurte notamment à un problème de moyens : «[L]orsque l'on veut construire un projet touristique dans le cadre d'un développement durable, il est important de se doter d'outil d'accompagnement opérationnel capable de prendre en compte les exigences théoriques d'une telle approche» (Torrente, 2003 : 11). L'écotourisme est lui aussi sujet à cette problématique. Des paramètres adaptés aux particularismes de cette forme de tourisme sont donc requis. Dans ce contexte, l'objectif de la présente analyse était de contribuer à leur caractérisation et à leur identification. À la différence des grilles génériques sur le développement durable, le résultat est ici une grille spécifique, adaptée aux particularismes de l'activité écotouristique.

Le présent article a par ailleurs permis d'identifier des critères de durabilité environnementale de l'écotourisme, dans une perspective de développement durable des territoires. Cette étude constitue une première étape visant à déterminer s'il est possible de paramétrer une démarche méthodologique d'opérationnalisation de la durabilité environnementale, lors de l'élaboration de projets écotouristiques sur un territoire 
Tableau 4: Critères spécifiques de la durabilité environnementale

\begin{tabular}{|c|c|}
\hline & Critères spécifiques \\
\hline \multirow{4}{*}{1.} & Respect de tous les traités, lois, normes et règlements relatifs à la protection de l'environnement \\
\hline & Constructions et matériaux conformes aux normes environnementales \\
\hline & Respect spécifique des statuts particuliers (aires protégées) \\
\hline & Certification de l'entreprise \\
\hline \multirow{2}{*}{2.} & Recours systématique à des évaluations environnementales et à des études d'impact \\
\hline & Évaluation de la capacité de charge/de support du site \\
\hline \multirow{7}{*}{3.} & Valorisation systématique de la responsabilité environnementale ; alphabétisation écologique \\
\hline & Existence de moyens d'encouragement des visiteurs à respecter le patrimoine et la culture locale \\
\hline & Formation et sensibilisation environnementale des employés \\
\hline & Sensibilisation environnementale de la population locale (communauté d'accueil) \\
\hline & Sensibilisation environnementale des visiteurs \\
\hline & Existence de programmes éducatifs spécifiquement destinés aux jeunes \\
\hline & Existence d'activités d'interprétation appropriées incluant de la documentation de sensibilisation à l'écoresponsabilité \\
\hline \multirow{5}{*}{4.} & Respect de la capacité de charge/de support du site \\
\hline & Consommation des ressources sous leur taux de renouvelabilité \\
\hline & Diversification de l'offre pour limiter la saturation de certains sites \\
\hline & Favorisation des petits groupes \\
\hline & Réduction des impacts des transports et de la mobilité \\
\hline 5. & Compatibilité des aménagements touristiques avec les composantes environnementales et patrimoniales du site \\
\hline \multirow{5}{*}{6.} & Conservation de l'intégrité des écosystèmes \\
\hline & Maintien de la diversité des écosystèmes et des espèces \\
\hline & Protection prioritaire des écosystèmes à haute valeur en conservation et des espèces menacées \\
\hline & Contrôle de l'importation de nouvelles espèces \\
\hline & Contrôle du prélèvement et de l'utilisation des espèces prélevées \\
\hline \multirow{10}{*}{7.} & Gestion des déchets : réduction, réutilisation (valorisation) et recyclage \\
\hline & Réduction de la consommation d'énergie \\
\hline & Conservation des ressources en eau \\
\hline & Gestion des eaux usées \\
\hline & Réduction de la pollution sonore \\
\hline & Réduction de la pollution visuelle \\
\hline & Réduction de l'érosion du site \\
\hline & Réduction de la pollution de l'air \\
\hline & Réduction de l'utilisation de produits chimiques nocifs pour l'environnement \\
\hline & Réduction des émissions de gaz à effet de serre (GES) \\
\hline \multirow{7}{*}{8.} & Respect des sites sensibles \\
\hline & Obtention d'un statut de protection pour le territoire d'exploitation \\
\hline & Système de traçabilité des actions \\
\hline & Utilisation d'infrastructures existantes et limitation du nombre des nouvelles \\
\hline & Construction de structures qui augmentent la durabilité des lieux \\
\hline & Utilisation rationnelle des matériaux \\
\hline & Utilisation systématique d'énergies renouvelables/alternatives \\
\hline \multirow{2}{*}{9.} & Existence de moyens permettant aux écotouristes de participer personnellement à l'effort de conservation \\
\hline & Mise en place de mesures compensatoires \\
\hline 10. & Existence de moyens de contrôle \\
\hline \multirow{2}{*}{11.} & Organisation d'évènements permettant d'interpréter le milieu naturel \\
\hline & Offre de services et outils de valorisation du patrimoine naturel \\
\hline
\end{tabular}

Source : compilation des auteurs.

TÉOROS, vol. 31, nº 2, p. 72-83 C 2012 
Tableau 5 : Grille pondérée des critères génériques de la durabilité écotouristique

\begin{tabular}{|c|c|c|}
\hline Volet du développement durable & Critères génériques & Nombre de textes \\
\hline Social & Bien-être des communautés d'accueil & 70 \\
\hline Social & Gouvernance participative & 66 \\
\hline Économique & Offre écotouristique ciblée et adaptée & 63 \\
\hline Environnemental & Préservation de l'intégrité géophysique du milieu (air, sol, eau) & 63 \\
\hline Économique & Marketing efficace et responsable & 62 \\
\hline Environnemental & Utilisation rationnelle des ressources & 58 \\
\hline Social & Valorisation de l'identité et du patrimoine culturel de la communauté d'accueil & 57 \\
\hline Économique & Valorisation des emplois & 55 \\
\hline Environnemental & Maintien de la pureté de l'environnement & 55 \\
\hline Social & Répartition large et équitable des bénéfices socio-économiques & 53 \\
\hline Économique & Rentabilité financière des projets écotouristiques & 48 \\
\hline Social & Respect des droits et structures sociales des communautés autochtones et traditionnelles & 47 \\
\hline Environnemental & Éducation relative à l'environnement & 44 \\
\hline Environnemental & Utilisation d'outils d'évaluation et de contrôle spécialisés & 38 \\
\hline Social & Inclusion des projets écotouristiques dans une stratégie de développement local & 33 \\
\hline Environnemental & Préservation de l'intégrité patrimoniale, de l'identité du site et des paysages & 32 \\
\hline Économique & Contrôle et suivi des activités/projets & 31 \\
\hline Environnemental & Préservation de l'intégrité écologique du site (biodiversité, écosystèmes) & 31 \\
\hline Social & Bien-être des visiteurs & 30 \\
\hline Économique & Coordination des agents économiques & 28 \\
\hline Environnemental & Mise en valeur du patrimoine naturel et culturel & 27 \\
\hline Économique & Maîtrise des outils et mécanismes de gestion & 25 \\
\hline Environnemental & Respect des obligations légales en matière environnementale & 22 \\
\hline Social & Respect de la communauté d'accueil par le promoteur & 22 \\
\hline Social & Adhésion du milieu d'accueil aux projets écotouristiques & 21 \\
\hline Économique & Démarche qualité transversale & 20 \\
\hline Économique & Communication efficace et éthique & 20 \\
\hline Économique & Planification et stratégie de développement & 18 \\
\hline Environnemental & Contribution proactive à la protection du milieu & 18 \\
\hline Environnemental & Gestion des interactions visiteurs/biodiversité (faune, flore) & 18 \\
\hline Économique & Promotion de la formation de la main-d'œuvre & 16 \\
\hline Économique & Implication des acteurs locaux & 13 \\
\hline Social & Sécurité des visiteurs & 13 \\
\hline Social & Connectivité civile et cohésion sociale & 10 \\
\hline Économique & Entrepreneuriat proactif et innovation & 2 \\
\hline
\end{tabular}

donné. Sur la base de l'identification des critères opérée ici, nous visons plus précisément la définition d'une ingénierie de projets écotouristiques favorisant, au stade de leur planification/conception, la valorisation du territoire hôte et la préservation de sa durabilité environnementale. L'outil final comportera, dans une approche de gestion de projet, une séquence intégrée de méthodes et un processus d'opérationnalisation des projets écotouristiques intégrant les critères spécifiques de la durabilité écotouristique, identifiés dans le présent article. Sa présentation fait l'objet d'une autre contribution.

\section{Références}

AEQ - Aventure Écotourisme Québec (2004) Étude sur la valeur économique de l'écotourisme et du tourisme d'aventure, Montréal : DBSF. 16 p.

AFIT - Agence Française de l'Ingénierie Touristique (2001) Piloter le tourisme durable dans les territoires et les entreprises, Paris : Atout France. 165 p.

BAHOLET, Sébastien (2008) Tourisme durable, Paris : Direction du tourisme. $13 \mathrm{p}$.

BEHNASSI, Mohamed (2008) «Tourisme durable : fondements, indicateurs et apport au développement des pays du sud», Revue de Droit et de Sciences Sociales, Université Ibn Zohr, nº 1, p. 96-120. 
BLAMEY, Russel K., (2001) «Principles of Ecotourism», The Encyclopedia of Ecotourism, Oxon, UK, New York : CABI Pub, p. 5-22.

BNQ - Bureau de normalisation du Québec (2003) «Norme NQ 9700060 Tourisme - Produits d'écotourisme», BNQ, <http://www.bnq. qc.ca/fr/certif/tourisme_norme.html>, consulté le 15 décembre 2011.

BOUTAUD, Aurélien (2005) «Le développement durable : penser le changement ou changer le pansement?». Thèse de Science et Génie de l'environnement, Saint-Étienne : École Supérieure des Mines de SaintÉtienne. $414 \mathrm{p}$.

BRETON, Jean-Marie (sous la direction de) (2006) Développement viable et valorisation environnementale, Pointe-à-Pitre : Éditions Karthala. 486 p.

BRODHAG, Christian (1999) Les enjeux de l'information en langue française sur le développement durable : conférence (Saint-Étienne, 5-9 juillet), Université d'Été Francophone développement durable et systèmes d'information, Saint-Etienne, Communications sans actes, $<$ http://wwwv1.agora21.org/univ-ete-fr/Christian-Brodhag.html>, consulté le 15 décembre 2011.

CAIRE, Gilles et Monique ROULET-CAIRE (2001) « Le tourisme peut-il être un élément de développement durable?», Les enjeux du développement durable, Orcades, p. 103-109.

CAZELAIS, Normand; Roger NADEAU et Gérard BEAUDET (1999) L'espace touristique, Québec: PUQ. 314 p.

CCE - Commission de coopération environnementale (2000) La promotion de l'écotourisme dans les aires naturelles de l'Amérique du Nord: Les étapes franchies, Montréal : CCE. 26 p.

CEBALLOS-LASCURÁIN, Hector (1987) Estudio de Prefectabilidad Socioeconomica del Turismo Ecologico y Anteproyecto Arquitectonico y Urbanistico del Centro del Turismo Ecologico de Sian Ka'an, Quintana Roo, étude réalisée pour SEDUE, Mexico, (non publié).

CEBALLOS-LASCURÁIN, Hector (1993) The IUCN Ecotourism Consultancy Programme, México : DF. 4 p.

CEBALLOS-LASCURAIN, Hector (1996) Tourism, Ecotourism and Protected Areas, Gland, Suisse : UICN. 301 p.

CERON, Jean-Paul et Ghislain DUBOIS (2002) «Les enjeux oubliés du tourisme durable», Cahiers Espaces, Paris : Éditions Touristiques Européennes, Hors-Série n ${ }^{\circ} 67$, p. 16-20.

CLARIMONT, Sylvie et Vincent VLÈS (2008) «L'intégration tardive et hésitante du tourisme dans le champ du développement durable», DANS Tourisme durable en montagne : entre discours et pratique, p. XVIIXXVII. La Plaine-Saint-Denis : AFNOR.

COCCOSSIS, Harry (sous la direction de) (2001) Définir, mesurer et évaluer la capacité de charge dans les destinations touristiques européennes, Athènes : Laboratoire de planification environnementale, Université de l'Égée. 58 p.

Comité des Régions (2011) Avis sur «L'Europe, première destination touristique au monde - un nouveau cadre politique pour le tourisme européen ", Journal officiel de l'Union européenne, (2011/C 104/03).

COUTURE, Maurice (2002) «L'écotourisme - un concept en constante évolution", Téoros, vol. 21, n 3, automne, p. 5-13.

CQRHT - Conseil Québécois des Ressources Humaines en Tourisme (1996) Diagnostic d'ensemble des ressources humaines en tourisme, orientations et plan d'action, Rapport final, Montréal : Groupe DBSF. $210 \mathrm{p}$.

DE JUAN ALONSO, José María (2010) Tourisme durable en méditerranée situation et perspectives, stratégies et actions, Malaga : IUCN. 99 p.

DENAIS, Laurent (2007) «Écotourisme, un outil de gestion des écosystèmes». Mémoire de maîtrise en écologie internationale, Sherbrooke : Université de Sherbrooke. 79 p.
DESMARAIS, Véronique (2007) «Un cadre d'évaluation du tourisme de nature durable en forêt publique québécoise». Mémoire de maîtrise, Faculté de foresterie, Québec : Université Laval. 115 p.

DESVIGNES, Claudine (sous la direction de) (2000) «Tourisme durable», Cahier Espaces $n^{\circ}$ 67, Editions ESPACES. 247 p.

DOWLING, Ross K. (2006) «Tourisme et communautés d'accueil, attitudes des communautés à l'égard des développements écotouristiques dans la région de Gascoyne, en Australie-Occidentale», DANS GAGNON et GAGNON (sous la direction de), L'écotourisme entre l'arbre et l'écorce, p. 109-141. Ste-Foy : PUQ.

GAGNON, Christiane (2008) «La construction historique du développement durable», Développement social, vol. 9, nº 2, p. 10-11.

GAGNON, Christiane (sous la direction de) (2012) Territoires durables en devenir, PUQ Numérique, 257 p., <http://www.puq.ca/catalogue/livres/ territoires-durables-devenir-1223.html $>$, consulté le 15 décembre 2011.

GAGNON, Christiane et Dominique LAPOINTE (2006) «Écotourisme et développement durable viable» DANS GAGNON et GAGNON (sous la direction de), L'écotourisme entre l'arbre et l'écorce, p. 13-42. Ste-Foy : PUQ.

GRENIER, Christophe (2000) Conservation contre nature. Les Îles Galapagos, Paris : IRD Éditions, Coll. «Latitudes $23 » .376$ p.

HONEY, Martha S. (1999) Ecotourism and Sustainable Development: Who Owns Paradise?, Washington, D.C : Island Press. 405 p.

KPMG Services Conseils (2010) Diagnostic - Tourisme nature, KPMG, $<$ http://www.tourisme.gouv.qc.ca/publications/media/document/ etudes-statistiques/Nature-master-2010-03-03.pdf >, consulté le 15 décembre 2011.

LALIBERTE, Michèle (2011) «L'écotourisme, trois décennies plus tard", Réseau de veille en tourisme, Chaire de tourisme TRANSAT ESGUQAM, <http://veilletourisme.ca/2011/04/04/1-ecotourisme-troisdecennies-plus-tard/>, consulté le 22 mars 2012.

LAURIOL, Jacques (2004) «Le développement durable à la recherche d'un corps de doctrine», Revue française de gestion, vol. 30, nº 152, p. 137 à 150

LEQUIN, Marie (2000) «Gouvernance en écotourisme : Développement durable, développement régional et démocratie participative». Thèse de doctorat en Études urbaines, Montréal : Université du Québec à Montréal. $523 \mathrm{p}$.

MACOUIN, Dominique et Isabelle PIERRE (2003) Le tourisme de nature, AFIT, Paris : Éditions Atout France. 43 p.

MARSAT, Jean-Bernard (2008) «Valorisation et gestion des aménités naturelles : Comment construire des stratégies d'alliance entre acteurs d'environnement et acteurs du tourisme?». Thèse de doctorat, Paris : AgroParisTech. 668 p.

OMT — Organisation mondiale du tourisme (2005) Tourisme et durabilité : Un aperçu général, Paris : Département du tourisme durable de l'Organisation mondiale du tourisme. $34 \mathrm{p}$.

PAUL, Alexandra; Olga Iga WIERCIOCH et Léo DAYAN (2007) Stratégies d'implémentation dans et par le tourisme, APREIS, octobre. 9 p. $<\mathrm{http} / / /$ www.apreis.org>, consulté le 10 février 2012.

PNUE — Programme des Nations unies pour l'environnement (1995) Charte du Tourisme Durable, Madrid : Conférence de Lanzarote. $4 \mathrm{p}$.

PNUE et OMT — Programme des Nations unies pour l'environnement et Organisation mondiale du tourisme (2007) «Définition simplifiée du comité français sur le tourisme durable», Définition du tourisme durable, Groupe de travail international sur le développement du tourisme durable (GTI-DTD), <www.veilleinfotourisme.fr>, consulté le 15 décembre 2011. 
PRISKIN, Julianna (2004) «Quand l'étiquette «écotourisme » est utilisée à toutes les sauces", Réseau de veille en tourisme, <http://veilletourisme. ca/2004/11/26/quand-letiquette-«ecotourisme»-est-utilisee-a-toutes-lessauces/>, consulté le 15 décembre 2011.

QUÉBEC (2005) Vers un tourisme durable, Politique touristique du Québec, Québec : BNQ. 40 p.

RIVARD, S. (2004) Vers une nouvelle Politique touristique du Québec, document de réflexion sur le développement durable du tourisme au Québec, Forum de l'industrie touristique, Québec : Tourisme Québec. 30 p.

RYAN, Chris (2002) «Equity, management, power sharing and sustainability - issues of the 'new tourism'», Tourism Management, vol. 23, no 1, p. 17-26.

SALAMÉRO, Sylvain (2010) «Spécialité politiques territoriales de développement durable». Master 2, Géographie et aménagement, Chicoutimi : Université du Québec a Chicoutimi. 97 p.

SOMMET MONDIAL DE L'ÉCOTOURISME (2002), Rapport final, Québec (Canada), 135 p.

TARDIF, Jonathan (2003) «Écotourisme et développement durable», VertigO, La revue électronique en sciences de l'environnement, Institut des sciences de l'environnement, Montréal : Université du Québec à Montréal, vol. 4, n 3, mis en ligne le 01 mai 2003, <http://vertigo.revues. org/4575>, consulté le 29 avril 2013.

TORRENTE, Pierre (2003) Le tourisme durable et la notion de projet : apports théoriques et méthodes, Toulouse : Université de Toulouse le Mirail. 29 p.
Tourisme Québec (2002) Nature et tourisme : L'écotourisme au Québec en 2002, octobre, BNQ, <http://www.tourisme.gouv.qc.ca/publications/ media/document/etudes-statistiques/eco_QC02.pdf> consulté le 15 décembre 2011.

Tourisme Québec (2007) Le Québec Grande nature — Plan intégré de l'expérience : Diagnostic et orientations, BNQ, <http://www.tourisme.gouv. qc.ca/publications/publication/quebec-grande-nature-plan-integreexperiencediagnostic-orientations-100.html? categorie $=39>$, consulté le 15 décembre 2011.

TRANQUARD, Manu (2010) Les impacts environnementaux du tourisme face au défi du développement durable, Chicoutimi : UQAC. 39 p.

TRANQUARD, Manu (2013) «Ingénierie des projets écotouristiques et durabilité environnementale». Thèse de doctorat en développement régional, Chicoutimi : Université du Québec à Chicoutimi. À venir.

VERNON, Jon; Stephen ESSEX et Kaja CURRY (2005) «Sustainable Rural Tourism Business Practice: progress and policy in South East Cornwall», DANS, HALL, D.; I. KIRKPATRICK et M. MITCHELL, Rural tourism and sustainable business, p. 323-352. Clevedon, Buffalo : Channel view publications.

VILLENEUVE, Claude (s. d.) Écotourisme et tourisme durable au Québec : Le défi de la crédibilité, Diaporama, Département des sciences fondamentales : Université du Québec à Chicoutimi. 29 images.

WEAVER, David B. (2001) The Encyclopaedia of Ecotourism, Wallingford : Cabi Publising. p. 657-666.

\section{APPROCHES MÉTHODOLOGIQUES EN TOURISME • APPEL À TEXTES}

Qu'elle soit qualitative ou quantitative, la recherche implique le recours à des techniques de collecte de données et d'analyses qui ont fait leurs preuves. Il arrive cependant que l'expérience touristique, par sa nature particulière (l'étude de phénomènes ou pratiques où certains acteurs sont en mode opérationnels alors que les prestataires sont en mode repos), nécessite une approche particulière. Considérant la nature multidisciplinaire du tourisme, et l'évolution rapides des expériences offertes, les chercheur(e)s sont ainsi confronté(e)s à des défis particuliers.

II y a un besoin réel de trouver des solutions adaptées aux problèmes engendrés par la recherche en tourisme et de développer des outils adaptés aux situations particulières du domaine. II est tout aussi important de jeter un regard critique sur les méthodologies employées jusqu'à présent en recherche touristique.

Téoros est à la recherche de textes consacrés à la méthodologie de recherche en tourisme, soit à propos de nouvelles approches, soit à propos de nouvelles façons de recourir aux méthodes de recherches existantes. Seront considérés les articles développés autour de discussions conceptuelles ou encore sur les applications de diverses méthodes. En plus de ces aspects, votre article peut porter sur:

- de nouvelles méthodes de recherche en tourisme selon les disciplines concernées par le phénomène;

- de nouvelles façons d'employer des méthodes de recherche conventionnelles en tourisme;
- des façons créatives de combiner les méthodes qualitative et quantitative;

- de nouvelles approches pour l'étude des questions contemporaines qui impliquent l'effort de communications entre les disciplines (comme, par exemple, la nature interdisciplinaire croissante des études en tourisme et le développement accéléré des études virtuelles).

Les auteur(e)s doivent faire parvenir un manuscrit rédigé préférablement en français présenté selon les règles de la revue, disponibles à l'url: http://teoros.revues.org/168. Les textes soumis, en format Word, doivent compter environ 7000 à 7500 mots et doivent comprendre un objectif (question) de recherche clairement énoncé; un descriptif de la méthodologie de recherche employée, et un volet théorique. Une étude de cas peut s'ajouter à ces éléments.

Chaque article doit inclure les nom et prénom de tous les auteurs, leur titre principal et leur affiliation (une seule), leur adresse électronique (courriel) et postale, un résumé de 150 à 200 mots maximum en français ainsi qu'une une liste des mots clés (maximum de 5). II n'y a pas de date limite pour soumettre un texte sur les approches méthodologiques: Téoros les reçoit en tout temps. Les propositions de textes doivent être adressées à la revue: teoros@ uqam.ca. Veuillez inscrire «Méthodologie» dans la ligne de sujet.

Au plaisir de vous lire dans nos pages! 